\title{
Prospective validation of a molecular prognostication panel for clival chordoma
}

\author{
Georgios A. Zenonos, MD, ${ }^{1}$ Juan C. Fernandez-Miranda, MD, ${ }^{1}$ Debraj Mukherjee, MD, MPH, ${ }^{1}$ \\ Yue-Fang Chang, PhD, ${ }^{1,2}$ Klea Panayidou, PhD, ${ }^{3}$ Carl H. Snyderman, MD, ${ }^{4}$ Eric W. Wang, MD, ${ }^{4}$ \\ Raja R. Seethala, MD, ${ }^{5}$ and Paul A. Gardner, MD' \\ ${ }^{1}$ Department of Neurosurgery, University of Pittsburgh Medical Center, Pittsburgh; ${ }^{2}$ Department of Biostatistics and \\ Epidemiology, University of Pittsburgh; ${ }^{3}$ Department of Statistics, Carnegie Mellon University, Pittsburgh; ${ }^{4}$ Department of \\ Otolaryngology, University of Pittsburgh Medical Center, Pittsburgh; and ${ }^{5}$ Department of Pathology, University of Pittsburgh \\ Medical Center, Pittsburgh, Pennsylvania
}

OBJECTIVE There are currently no reliable means to predict the wide variability in behavior of clival chordoma so as to guide clinical decision-making and patient education. Furthermore, there is no method of predicting a tumor's response to radiation therapy.

METHODS A molecular prognostication panel, consisting of fluorescence in situ hybridization (FISH) of the chromosomal loci $1 p 36$ and 9p21, as well as immunohistochemistry for Ki-67, was prospectively evaluated in 105 clival chordoma samples from November 2007 to April 2016. The results were correlated with overall progression-free survival after surgery (PFSS), as well as progression-free survival after radiotherapy (PFSR).

RESULTS Although Ki-67 and the percentages of tumor cells with 1q25 hyperploidy, $1 \mathrm{p} 36$ deletions, and homozygous 9p21 deletions were all found to be predictive of PFSS and PFSR in univariate analyses, only 1 p36 deletions and homozygous 9 p21 deletions were shown to be independently predictive in a multivariate analysis. Using a prognostication calculator formulated by a separate multivariate Cox model, two 1 p36 deletion strata $(0 \%-15 \%$ and $>15 \%$ deleted tumor cells) and three 9p21 homozygous deletion strata ( $0 \%-3 \%, 4 \%-24 \%$, and $\geq 25 \%$ deleted tumor cells) accounted for a range of cumulative hazard ratios of 1 to 56.1 for PFSS and 1 to 75.6 for PFSR.

CONCLUSIONS Homozygous 9p21 deletions and 1p36 deletions are independent prognostic factors in clival chordoma and can account for a wide spectrum of overall PFSS and PFSR. This panel can be used to guide management after resection of clival chordomas.

https://thejns.org/doi/abs/10.3171/2018.3.JNS172321

KEYWORDS chordoma; prognosis; genetics; radiation; surgery; oncology

$\mathrm{W}$ HILE the average survival after treatment of skull base chordomas, which involves surgery with or without adjuvant radiation therapy, is approximately 7.7 years, their clinical course has been found to be extremely variable. ${ }^{714}$ Unfortunately, there are no reliable means of predicting this diverse behavior. Not only is there no validated molecular prognostication panel available, but even histology has been proven to be unreliable. ${ }^{6,26}$ As a result, the World Health Organization has no formal grading system for these tumors, and clinical decisions about early reoperation, adjuvant radiation therapy, or observation are made largely empirically.

The ability to prognosticate is especially important for skull base chordomas, as their close relationship to critical neurovascular structures rarely allows for cura- tive extirpation with negative margins. Consequently, even after gross-total resection, the biology of infiltrating microscopic remnants most likely dictates progression-free survival (PFS). Because adjuvant radiation therapy to address these microscopic remnants can also be associated with significant morbidity, its routine use is not universally espoused. ${ }^{21,26}$ To add to the uncertainty, there are no data to help determine the likelihood of a specific chordoma responding to radiation therapy.

Two molecular markers of special interest in chordoma are losses of the 1p36 and 9p21 chromosomal loci. Frequent deletions of $1 \mathrm{p} 36$ have been recognized early in chordoma, , 513,20,22 although the biological and clinical significance of this finding was never elucidated. Previous work identified a possible association of $9 p$ loss of

ABBREVIATIONS FISH = fluorescence in situ hybridization; PFS = progression-free survival; PFSR = PFS after radiotherapy; PFSS = PFS after surgery. SUBMITTED September 25, 2017. ACCEPTED March 12, 2018.

INCLUDE WHEN CITING Published online June 15, 2018; DOI: 10.3171/2018.3.JNS172321. 
heterozygosity (p16/CDKN2A) with a worse prognosis, but because of the limited number of samples, the results were not internally consistent, and the clinical repercussions were difficult to quantify. ${ }^{13}$ Based on this information, fluorescence in situ hybridization (FISH) of 1p36 and 9p21 as well as immunohistochemistry for Ki-67 was prospectively evaluated over the past decade in an attempt to validate a clinically applicable prognostication panel. Using a separate analysis, we also devised a prognostication scheme for tumors undergoing radiation therapy. In this report, we present our results and propose a clinical management algorithm based on our findings.

\section{Methods}

\section{Sample Acquisition and Histology}

This study was performed with the approval of the institutional review board of the University of Pittsburgh. Clival chordoma specimens were obtained from surgical procedures performed at our institution from November 2007 to April 2016, and were prospectively analyzed. Both primary and recurrent tumors were included. The basic demographics are presented in Table 1. The pathologic diagnosis of chordoma was confirmed by 2 observers on $\mathrm{H}$ \& E slides, as well as by immunohistochemical analysis (brachyury, S100B, cytokeratins, vimentin, and epithelial membrane antigen). Since there is no formal pathological grading system available other than classifying the tumor as conventional or chondroid, the pathology reports often commented on potential atypical or dedifferentiation features associated with the tumor (e.g., areas of increased cohesiveness and cellularity, nuclear or nucleolar atypia, unusual staining patterns, or focal areas of dedifferentiation, among others).

\section{Follow-Up Practices and Radiation}

All patients underwent baseline MRI after surgery, at 3 and 9 months postoperatively, and then yearly thereafter unless there were radiographic or clinical concerns. All MRI studies were obtained with our skull base protocol. For patients undergoing follow-up imaging at other institutions, specific instructions were given for these MRI studies to comply with our standards. This protocol includes thin-cut T2-weighted images in the axial, coronal, and sagittal planes; thin-cut volumetric contrasted SPGR (spoiled gradient recalled acquisition in steady state) sequences in all 3 planes; and T1-weighted sequences without contrast. Each of these MRI studies was evaluated at our comprehensive skull base conference, and disease progression was determined after consensus of all participants, including skull base neurosurgeons, otolaryngology surgeons, and neuroradiologists specializing in skull base pathology, as well as neuro-ophthalmologists and oculoplastic surgeons. The preoperative characteristics of the tumor (e.g., anatomical structures and compartments involved, degree of enhancement, homogeneity, and degree of T2 hyperintensity), as well the details pertaining to the surgery (e.g., difficult areas to access), are all taken into consideration when evaluating for disease progression.

Radiation in the form of proton-beam radiotherapy (n $=67)$ or stereotactic radiosurgery $(n=9)$ was adminis-
TABLE 1. Basic demographics

\begin{tabular}{lcc}
\hline & PFSS $(n=105)$ & PFSR $(n=76)$ \\
\hline Mean age (SD), yrs & $47(17.3)$ & $45(20.1)$ \\
\hline Male sex & $66 \%(n=69)$ & $67 \%(n=51)$ \\
\hline Recurrent tumors & $55 \%(n=58)$ & $53 \%(n=40)$ \\
\hline
\end{tabular}

tered, either as an adjuvant treatment after surgery (for microscopic or gross residuals) or for recurrent tumors. In general, adjuvant radiation treatment was recommended if there was significant concern for residual disease, although the patient's age and comorbidities as well as the desire to have children were taken into consideration when discussing this with each individual patient.

\section{FISH Protocol}

FISH protocols were similar to those described previously. ${ }^{20}$ Formalin-fixed, paraffin-embedded tissue microarray sections were mounted and serially sectioned at 5-mm intervals. H \& E sections were used to determine the area of the tissue to be targeted for analysis. FISH slides were deparaffinized in xylene twice for 10 minutes, dehydrated twice with $100 \%$ ethanol, and then pretreated using the Vysis paraffin pretreatment kit (Abbott Molecular Inc.). Slides were digested for 18 minutes in protease solution $(0.5 \mathrm{mg} / \mathrm{ml})$ at $37^{\circ} \mathrm{C}$. For $1 \mathrm{p} 36$, hybridization was performed using a spectrum orange-labeled probe for 1 p36 and a spectrum green-labeled control probe for 1q25 as an index of chromosome 1 (Vysis dual-color probe set, LSI 1p36/LSI 1p25, Vysis). For 9p21, hybridization was performed using a spectrum orange-labeled probe for CDKN2A (p16) and a spectrum green-labeled chromosome 9 centromeric probe (CEP 9) as an index of chromosome 9 (Vysis). The target slide and probe were codenatured at $95^{\circ} \mathrm{C}$ for 8 minutes and incubated overnight at $37^{\circ} \mathrm{C}$ in a humidified chamber. Posthybridization washes were performed using $2 \times \mathrm{SSC} / 0.3 \%$ Igepal at $72^{\circ} \mathrm{C}$ for 2 minutes. The slides were air dried in the dark and counterstained with DAPI (Vysis). Analysis was performed using a Nikon Optiphot-2 (Nikon Inc.) and Quips Genetic Workstation equipped with a Chroma Technology filter set with single band exciters for spectrum orange, fluorescein isothiocyanate, and DAPI (ultraviolet $360 \mathrm{~nm}$ ) (Vysis). Only individual and well-delineated cells were scored. Overlapping cells were excluded from the analysis. At least 80 nuclei were counted in each case. For 1p36, the pathology report included the total percentage of cells with 1p36 deletion and the percentage of cells with 1q25 hyperploidy (as an index of chromosome 1 hyperploidy), as well as the ratio of 1 p36 to $1 \mathrm{q} 25$ signals in the counted nuclei (1p36/1q25 ratio). For $9 \mathrm{p} 21$, the report included the percentages of cells with homozygous deletions, heterozygous deletions, complex deletions (such as deletions in hyperploid cells), and the total percentage of cells with any deletion. In addition, the report included the percentage of cells with CEP 9 monoploidy and CEP 9 hyperploidy (as an index of chromosome 9 monoploidy and hyperploidy, respectively), as well as the 9p21 to CEP 9 signal ratio in the counted nuclei (9p21/CEP ratio). 
Notably, harvesting of the samples during surgery was performed with caution so as to only include a clean specimen of noncauterized tumor, avoiding contamination from other tissue (such as dura and connective tissue). As such, the percentages of cells with a reported finding on FISH were highly representative of the population of tumor cells with the respective finding.

\section{Immunohistochemistry Protocol for Ki-67}

Formalin-fixed, paraffin-embedded tissues were cut at $6 \mu \mathrm{m}$ and incubated with antibodies for Ki-67 (dilution 1:100, MIB-1, Dako). Staining was visualized using the ImmPRESS (Vector Laboratories) detection system with 2-diaminobenzidine as the substrate chromogen. Each antibody was scored manually between 2 observers. The Ki-67 proliferation index was estimated as the percentage of positive tumor nuclei. The reported percentage represented the area of the analyzed tumor with the highest proliferation rate.

\section{Data Analysis}

The primary end point was PFS. The Kaplan-Meier method was used to calculate the overall PFS after surgery (PFSS). As such, PFSS was determined as the time period between surgery and either the time of recurrence or the last time the patient was censored, regardless of subsequent radiation therapy. A separate analysis was performed for 76 tumors receiving radiation after surgery, either as adjuvant therapy or for recurrent disease. For these patients, PFS after radiotherapy (PFSR) was calculated as the time period between radiation therapy and either the time of recurrence or the last time the patient was censored. For the purposes of calculations in this group, we used the molecular profile obtained from the resection preceding the radiation treatment.

Overall, of the 105 tumors used in the overall PFSS calculations, 103 tumors had Ki-67 data, 100 tumors had 1p36 FISH data, and 99 tumors had 9p21 FISH data (97 tumors had a complete set of data, 4 tumors had only Ki67 data, 2 tumors had Ki-67 and 1p36 data, 1 tumor only had 9p21 data, and 1 had $1 \mathrm{p} 36$ and 9p21 data).

Of the 76 tumors used for the PSFR calculations, 74 tumors had Ki-67 data, 49 had 1p36 data, and another 49 had 9p21 FISH data (47 tumors had a complete set of data, 1 tumor had Ki-67 and 1p36 data, 1 tumor had 1p36 and 9p21 data, 1 only had 9p21 data, and 26 tumors only had Ki-67 data). Fifty-four tumors were the same as the ones used for the PFSS calculations, but 22 extra tumors with only Ki-67 data were added to increase the power of the PFSR calculations with Ki-67.

Given that chordomas have been shown to be polyclonal tumors, ${ }^{16}$ the molecular indices were initially considered as continuous variables so as to evaluate for graded effects on prognosis, depending on the percentage of tumor cells bearing a specific molecular aberrancy.

Statistical analyses were performed using IBM SPSS (version 24, IBM Corp.). Bootstrapping with 1000 resamplings was used for all analyses to improve the accuracy and precision of all estimators under the Cox proportional hazards model.

\section{Results}

\section{Univariate Screening Analysis}

An initial screening analysis was performed using a univariate Cox proportional hazards model, which included all the molecular indices as continuous variables. Based on this analysis, the same molecular indices were predictive of PFSS and PFSR: Ki-67, total percentage of cells with 9p21 deletion, percentage of cells with homozygous 9p21 deletions, 9p21/CEP ratio, percentage of cells with $1 \mathrm{p} 36$ deletion, the $1 \mathrm{p} 36 / 1 \mathrm{q} 25$ ratio, and the percentage of cells with 1q25 hyperploidy. The detailed results are presented in Supplementary Table 1.

\section{Molecular Index Stratification}

In order to increase the clinical relevance of our findings, we proceeded to stratify select molecular markers. The molecular markers chosen were found to be significant in our univariate analysis above, but were also more direct and intuitive to interpretation. As such, the Ki-67, the percentage of cells with homozygous 9p21 deletions, the percentage of cells with $1 \mathrm{p} 36$ deletion, and the percentage of cells with 1q25 hyperploidy were stratified. Using Kaplan-Meier log-rank analyses, a variety of cutoffs were trialed to define the strata that affected PFS in the most meaningful way. For $\mathrm{Ki}-67$ and the percentage of cells with homozygous 9p21 deletions, we were able to separate the indices into 3 strata. For the percentage of cells with $1 \mathrm{p} 36$ deletions and 1q25 hyperploidy, we were only able to generate 2 meaningful strata, as there appeared to be an inflection point around the 15th percentile of tumor cells involved.

The generated Kaplan-Meier survival curves for each stratum of the molecular indices are shown in Figs. 1 and 2 (PFSS and PFSR, respectively). Long rank statistics revealed statistically significant differences between each of the molecular indices' strata (with the single exception of the Ki-67 strata 4\%-10\% and > 10\% for PFSR in Fig. 2). A Cox analysis using the strata as categorical variables was used to define the hazard ratios between the strata.

\section{Multivariate Analyses}

The stratified molecular indices (Ki-67, percentage of cells with homozygous 9p21 deletions, percentage of cells with 1p36 deletion, and the percentage of cells with 1q25 hyperploidy) were entered into a multivariate Cox proportional hazards model to determine if they were independently predictive of PFS. This was performed for both PFSS and PFSR. As seen in Table 2, when all the molecular indices were entered into the statistical model, only the percentages of cells with $1 \mathrm{p} 36$, and homozygous 9 p21 deletions were found to be independently predictive of progression-free survival. Interestingly, Ki-67, a marker found to be strongly predictive in our univariate analyses, was not independently predictive when the other indices were accounted for, although in the PFSR analysis, a Ki$67>10 \%$ approached significance.

The median and 36-month PFSS and PFSR associated with combinations of $1 \mathrm{p} 36$ and 9p21 deletion strata are shown in Table 3 . The results of a second multivariate analysis, now only considering the $1 \mathrm{p} 36$ and 9p21 deletion 

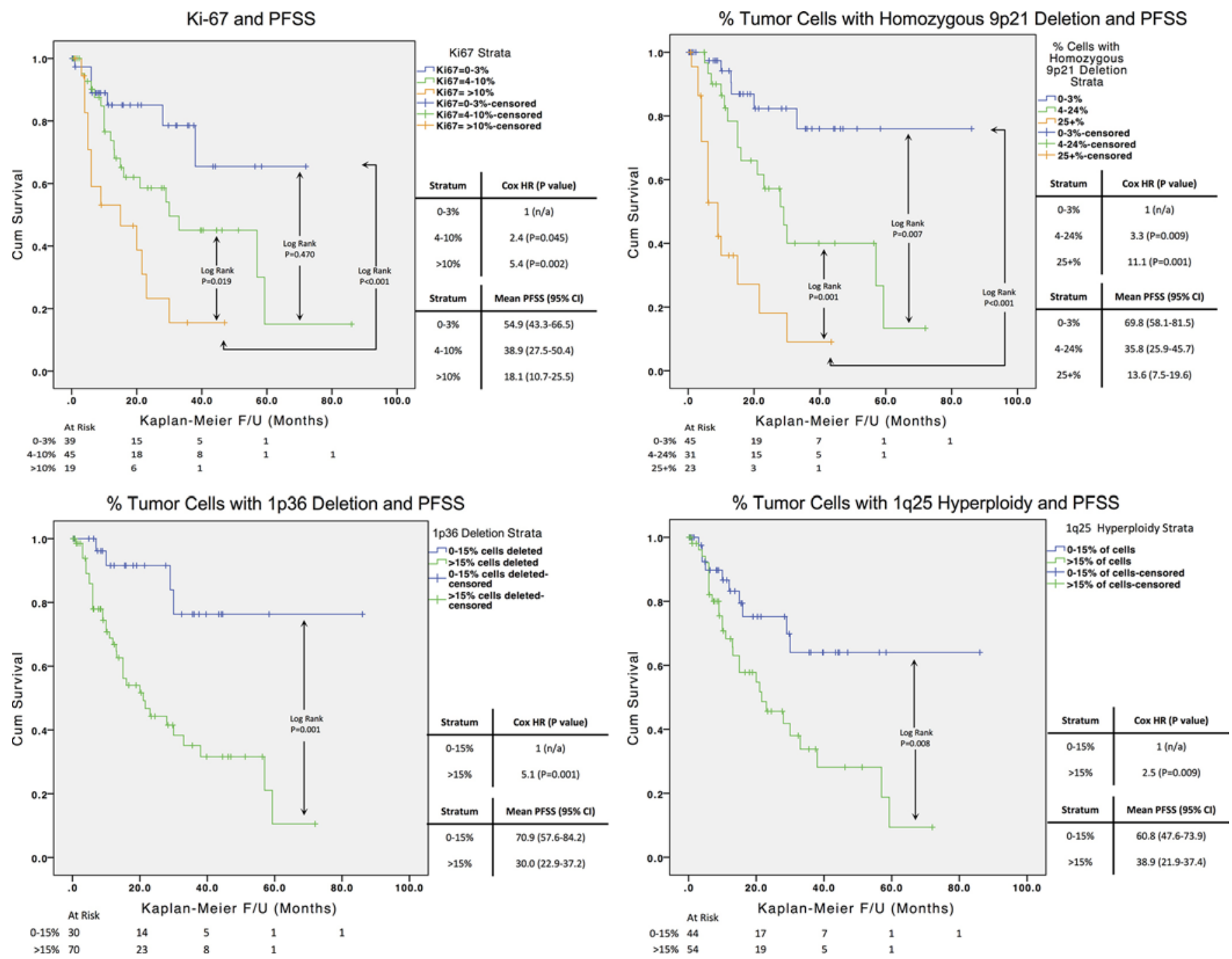

FIG. 1. Kaplan-Meier curves showing overall PFSS for each of the molecular index strata. Log-rank $p$ values showing the statistical significance of the differences among the survival curves of each panel. Cox proportional hazard ratios of each stratum compared to the first are shown next to each panel. The mean PFSS in months and the $95 \% \mathrm{Cl}$ for each stratum are also provided. PFSS refers to the interval between surgery and either the time of recurrence or the last time the patient was censored, whichever came first. $\mathrm{n} / \mathrm{a}=$ not applicable. Figure is available in color online only.

strata (the only factors found to be independently predictive of PFS in our aforementioned multivariate analysis), are shown in Table 4. This table can be used as a prognostication calculator with which a cumulative hazard ratio can be estimated by multiplying the individual hazard ratios of the multivariate analysis. As such, two $1 \mathrm{p} 36$ deletion strata $(0 \%-15 \%$ and $>15 \%$ deleted cells) and three 9 p21 homozygous deletion strata $(0 \%-3 \%, 4 \%-24 \%$, and $\geq 25 \%$ deleted cells) accounted for a very wide range of cumulative hazard ratios (1-56.1 for PFSS and 1-75.6 for PFSR).

The generated strata of $1 \mathrm{p} 36$ and $9 \mathrm{p} 21$ were subsequently categorized into 3 groups (A, B, and C) as seen in Fig. 3. The Kaplan-Meier survival curves of PFSS and PFSR are shown for each of these groups.

For each of the groups, a Cox proportional hazards analysis was performed to determine whether there was a statistically significant difference in PFS after surgery for tumors that received radiation therapy or not. In all of the groups, there was a homophonous trend toward better survival for tumors that did receive radiation therapy. However, this did not reach statistical significance for any of the groups (group A: HR 46.2, $\mathrm{p}=0.319$; group B: HR $1.66, p=0.291$; group C: HR 1.25, $p=0.683$ ). For the entire group, the HR was 1.39 (increased risk of recurrence for nonradiated tumors), but again this was not statistically significant $(\mathrm{p}=0.338)$.

\section{Correlation of Chordoma Histology With Molecular Index Profile}

Six samples were characterized as chondroid chordomas. These did not have any significant differences with the rest of the cohort with regard to the mean Ki-67 or percentage of cells with homozygous 9p21 deletions, $1 \mathrm{p} 36$ deletions, or 1q25 hyperploidy (independent sample Mann-Whitney U-test $\mathrm{p}$ values of $0.67,0.98,0.40$, and 

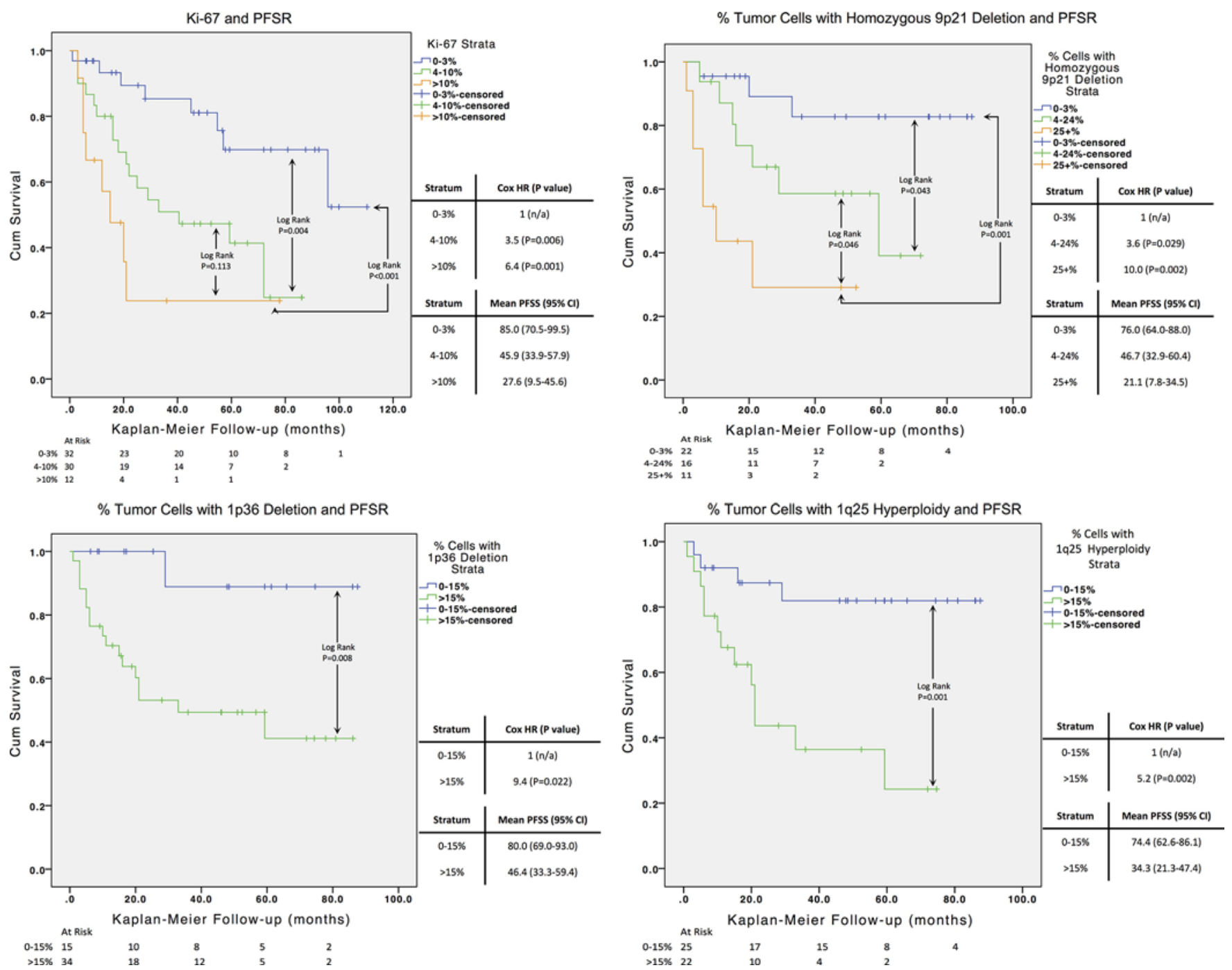

FIG. 2. Kaplan-Meier curves showing PFSR for each of the molecular index strata. Log-rank $p$ values show the statistical significance of the differences among each survival curve. Cox proportional hazard ratios of each stratum compared to the first are shown next to each panel. The mean PFSR in months and the $95 \% \mathrm{Cl}$ for each stratum are also provided. PFSR refers to the time interval between radiation and either the time of recurrence or the last time the patient was censored, whichever came first. Figure is available in color online only.

0.41 , respectively). Furthermore, a Kaplan-Meier log-rank test did not reveal any significant differences with regards to the overall PFSS $(\mathrm{p}=0.76)$, which was consistent with prior reports. ${ }^{6}$

Atypical/dedifferentiation features were diverse and difficult to quantify for calculation purposes. However, a notable observation was that of the 92 tumors reported as conventional or chondroid without any atypical features noted, only 17 had the most benign molecular profile (1p36 deletions, $0 \%-15 \%$; and $9 \mathrm{p} 21$ deletions, $0 \%-3 \%$ ). None of the remaining tumors with any reported atypical pathological features was associated with this favorable profile.

\section{Discussion}

Since the establishment of the Chordoma Foundation (www.chordomafoundation.org) in 2007, there have been coordinated efforts to improve our understanding of these tumors. Chordomas, however, remain an elusive disease for which little has been translated into clinical applications. ${ }^{4}$

Factors that have been associated with a potentially worse prognosis in chordoma include expression of pAKT, ${ }^{10}$ Raf- $1,{ }^{28}$ Survivin,,${ }^{8}$ iNOS, ${ }^{2,3}$ Ki-M1p, ${ }^{3}$ VEGFR-2, ${ }^{3}$ and mTOR, ${ }^{9}$ as well as overexpression of CDK4, ${ }^{27}$ brachyury, ${ }^{15}$ PDGFR-a, ${ }^{1}$ EGFR, ${ }^{1}$ c-MET, ${ }^{1}$ SPHK $1,{ }^{28}$ and p53. ${ }^{27}$ Furthermore, epigenetic dysregulation with miR$1^{11}$ or miR-1237-3p downregulation, ${ }^{29}$ or overexpression of miR-140-3 $\mathrm{p}^{30}$ or miR-155 $5^{23}$ has been associated with a potentially worse prognosis. With a disease incidence not exceeding 1 per million annually, 7,14 chordoma-related research has been very challenging and, unfortunately, the limited number of samples in most studies precluded any meaningful quantification of outcome measures or clinical applicability of any results. In addition, most reports 
TABLE 2. Multivariate Cox proportional hazards survival analysis for overall PFSS and PFSR

\begin{tabular}{|c|c|c|c|c|c|}
\hline \multirow[b]{2}{*}{ Molecular Index } & \multirow[b]{2}{*}{ Stratum } & \multicolumn{2}{|l|}{ PFSS } & \multicolumn{2}{|l|}{ PFSR } \\
\hline & & HR (compared w/ 1st stratum) & $p$ Value & HR (compared w/ 1st stratum) & $\mathrm{p}$ Value \\
\hline \multirow{3}{*}{ Ki-67 } & $0-3 \%$ & & & & \\
\hline & $4-10 \%$ & 2.08 & 0.20 & 3.61 & 0.13 \\
\hline & $>10 \%$ & 2.37 & 0.19 & 5.23 & 0.09 \\
\hline \multirow{3}{*}{ Homozygous 9p21 deletion } & $0-3 \%$ & & & & \\
\hline & $4-24 \%$ & 2.87 & $0.03^{*}$ & 4.91 & $0.02^{*}$ \\
\hline & $\geq 25 \%$ & 10.33 & $0.001^{*}$ & 6.80 & $0.02^{*}$ \\
\hline \multirow{2}{*}{$1 p 36$ deletion } & $0-15 \%$ & & & & \\
\hline & $>15 \%$ & 3.98 & $0.04^{*}$ & 5.32 & $0.047^{*}$ \\
\hline \multirow{2}{*}{ 1q25 hyperploidy } & $0-15 \%$ & & & & \\
\hline & $>15 \%$ & 1.12 & 0.83 & 1.29 & 0.76 \\
\hline
\end{tabular}

Each stratum was considered as a categorical variable.

${ }^{*}$ Statistically significant.

are based on sacral chordomas, and, given some evidence supporting skull base chordomas as being biologically distinct from their sacral counterparts, ${ }^{27}$ the generalizability of these studies to clival chordomas is questionable.

Below, we briefly review the biology related to the described prognostication panel, propose a clinically applicable management algorithm based on our results, and discuss the logistics and limitations of our findings.

\section{1p36 and 9p21 Loci, and Ki-67}

$\mathrm{Ki}-67$, as an index of mitotic activity, is one of the most widely used gauges of tumor aggressiveness. Indeed, $\mathrm{Ki}$ 67 was previously shown to have prognostic significance in chordoma, with values above $5 \%$ being associated with a shorter PFS. ${ }^{13,15}$ Our results not only corroborate these findings but also show a clear graded shortening of both PFSS and PFSR with progressively higher Ki-67 values.

Interestingly, when accounting for $1 \mathrm{p} 36$ and homozygous 9p21 deletions in our multivariate analysis, Ki-67 was no longer independently predictive of PFS. Although there are presumably multiple genetic aberrations responsible for dysregulation of the cell cycle in chordoma, the fact that Ki-67 was rendered insignificant in the multivariate analysis suggests that $1 \mathrm{p} 36$ and $9 \mathrm{p} 21$ deletions are responsible for a significant proportion of the tumor's phenotypic mitotic activity. Of course, this is only a statistical observation, and further investigation with functional tests (such as with overexpression or knockdown studies in a cell system) is needed to determine if this holds true in vivo.

Losses of the 9p21 chromosomal locus by FISH have been used as a surrogate of deletions of the tumor suppressor genes coding for CDKN2A $/ \mathrm{p}^{16^{\text {Ink4a }}}$, p14 ${ }^{\mathrm{ARF}}$, and $\mathrm{p} 15^{\text {Ink4b }}$, all of which are products of alternative splicing of the same locus, and all of which have critical roles in the regulation of the cell cycle. ${ }^{24}$ Deletions of CDKN2A/p16 $6^{\text {Ink4a }}$ leading to cell cycle dysregulation have been well described in a variety of other tumors, such as melanoma, esophageal, and gastric cancers, among others. ${ }^{24}$ Such deletions were reported to also occur frequently in chordoma, but their biological significance remained unclear. ${ }^{19,27}$ A retrospective study on 28 chordomas had identified a potential association of $9 p$ polymerase chain reaction-based loss of heterozygosity with worse prognosis, but the association with deletions based on 9p21 FISH only approached significance. ${ }^{13}$ The current study not only validates the robust association between 9p21 deletions and worse prognosis, but also shows a clear graded relationship between the percentage of tumor cells with homozygous 9p21 deletions and PFS. This graded effect also underlines the polyclonality of these tumors, which may have significant implications for potential experimental chemotherapeutic treatments. Interestingly, new chemotherapeutic agents modulating the p16 pathway, such as palbociclib (a CDK4/6 inhibitor), have shown preclinical efficacy in chordoma. ${ }^{25}$ As such, patients with tumors

TABLE 3. The 36-month and median overall PFSS and PFSR

\begin{tabular}{|c|c|c|c|c|c|c|c|}
\hline & Homozygous 9p21 Deletion & No. of Pts & 36-Mo PFSS & Median PFSS (mos) & No. of Pts & 36-Mo PFSR & Median PSFR (mos) \\
\hline \multirow{3}{*}{$1 p 36$ deletion $0-15 \%$} & $0-3 \%$ & 17 & $92 \%$ & 86.0 & 8 & $100 \%$ & 87.0 \\
\hline & $4-24 \%$ & 10 & $69 \%$ & 39.0 & 5 & $75 \%$ & 65.0 \\
\hline & $\geq 25 \%{ }^{*}$ & 3 & $60 \%$ & 43.0 & 2 & $100 \%$ & 47.0 \\
\hline \multirow{3}{*}{$1 p 36$ deletion $>15 \%$} & $0-3 \%$ & 27 & $72 \%$ & 51.0 & 13 & $72 \%$ & 86.0 \\
\hline & $4-24 \%$ & 21 & $37 \%$ & 23.6 & 11 & $52 \%$ & 59.2 \\
\hline & $\geq 25 \%$ & 20 & $0 \%$ & 6.7 & 9 & $18 \%$ & 6.7 \\
\hline
\end{tabular}

Pts = patients.

All of the strata combinations of $1 p 36$ deletions and 9p21 homozygous deletions are shown.

* Limited number of tumors in this group. 
TABLE 4. Cumulative hazard ratio prognostication calculator

\begin{tabular}{|c|c|c|c|c|c|c|c|}
\hline & 1p36 Deletion Status & HR "A" & $p$ Value & 9p21 Deletion Status & $H R$ "B" & $p$ Value & Cumulative HR \\
\hline \multirow{3}{*}{ PFSS } & $0-15 \%$ & 1 & & $0-3 \%$ & 1 & & \multirow{3}{*}{$H R$ "A" × HR "B" } \\
\hline & \multirow{2}{*}{$>15 \%$} & \multirow{2}{*}{5.1} & \multirow{2}{*}{0.003} & $4-24 \%$ & 2.5 & 0.039 & \\
\hline & & & & $\geq 25 \%$ & 11.0 & 0.001 & \\
\hline \multirow{3}{*}{ PFSR } & $0-15 \%$ & 1 & & $0-3 \%$ & 1 & & \multirow{3}{*}{$H R$ "A" $\times$ HR "B" } \\
\hline & \multirow{2}{*}{$>15 \%$} & \multirow{2}{*}{8.4} & \multirow{2}{*}{0.025} & $4-24 \%$ & 3.1 & 0.046 & \\
\hline & & & & $\geq 25 \%$ & 9.0 & 0.001 & \\
\hline
\end{tabular}

Based on a multivariate Cox proportional hazards survival analysis considering only 1 p36 and homozygous 9 p21 deletions, cumulative hazard ratios can be calculated for both overall PFSS and PFSR by multiplying hazard ratio "A" (corresponding to the hazard ratios of the 1p36 deletion strata) by hazard ratio "B" (corresponding to the hazard ratios of the $9 p 21$ deletion strata). The estimated cumulative hazard ratios express how much more likely the tumor in question is to recur when compared with a tumor with baseline strata for both markers ( $0 \%-15 \%$ cells with $1 \mathrm{p} 36$ deletions and $0 \%-3 \%$ cells with homozygous $9 \mathrm{p} 21$ deletions).

harboring high percentages of cells with homozygous $9 \mathrm{p} 21$ deletions may be better candidates for newly initiated clinical trials testing similar agents in patients with clival chordoma (e.g., NCT03110744, clinicaltrials.gov). Given that no pharmacotherapy has proven to be helpful in chordoma so far, the establishment of an efficacious drug is a crucial priority (https://www.chordomafoundation.org/latest-updates/ top-10-takeaways-fifth-international-chordoma-researchworkshop/).

Deletions of $1 \mathrm{p} 36$ have been well described in a wide variety of malignancies, and were often linked to a more aggressive phenotype. In addition, similar deletions have been implicated in various premalignant lesions and/or early stages of cancer, suggesting an early role in carcinogenesis ${ }^{4,12}$ Despite the relative frequency of this genetic aberration in cancer, the quest for the exact tumor suppressor gene(s) associated with this locus has been challenging. Of the 52 total genes encompassed within the $1 \mathrm{p} 36$ region, the ones that have been shown to have tumorigenic potential when reduced in dosage by genomic copy number loss or other mechanisms are CHD5, CAMTA1, KIFIB, CASZ1, and $m i R-34 a a^{4,12} \mathrm{~A}$ discussion of the complicated processes with which reduced expression of these genes might lead to carcinogenesis is beyond the scope of the current article and is detailed elsewhere.,12 However, it has been shown that coordinated loss of the 1p36 genes and their downstream effectors is a powerful tumorigenic event, even in haploinsufficient cells. ${ }^{12}$ Nevertheless, the significance of these deletions remained unclear for chordoma, as none of the prior studies evaluating correlations with clinical outcomes was adequately powered to reach statistical conclusions. ${ }^{5,13,20}$ The current report shows a robust association of 1 p36 deletions with a shorter PFS in chordoma. Notably, a dose-effect relationship was not as clear-cut as with 9p21 deletions and $\mathrm{Ki}-67$, as there appeared to be an inflection point at the 15 th percentile of $1 \mathrm{p} 36$ deletions. The exact reason for this phenomenon is not clear, but it could reflect a distinct pathogenesis between tumors with and those without 1p36 deletions. Although 1q25 hyperploidy was associated with worse prognosis in our univariate analyses, given that it was rendered insignificant in the multivariate analysis, its biological importance remains unclear.

Collectively, the current report points to a strong role of 1p36 and 9p21 deletions in the pathobiology of chordomas. Further elucidation of the exact mechanisms involved could yield much-needed druggable targets. More impor- tantly, these findings have significant clinical repercussions, which could be applicable immediately.

\section{Clinical Applicability of Results}

With no proven chemotherapeutic options available, the current standard of care for skull base chordomas involves maximal safe resection with or without adjuvant radiation therapy. However, due to our poor ability to prognosticate, decisions about further treatment after initial resection are largely made empirically. Gross-total resections have been the single most important factor to influence PFS..$^{17,18,26}$ Nonetheless, when complete resection is not possible through a single approach or without the risk of significant approach-related morbidity (e.g., in the setting of neurovascular encasement or multifocal recurrence), the surgeon has no means of quantifying the risk/ benefit ratio of recommending further surgery to achieve a complete resection (e.g., procedures with increased risk of sacrificing a cranial nerve, sacrificing or bypassing major vasculature). In addition, although adjuvant radiation therapy, usually in the form of proton-beam radiotherapy, has been found to improve PFS, its potential for significant morbidity and the absence of any data to help predict response to radiotherapy have led to a wide variability in practices. ${ }^{21,26}$ As a result, some practitioners recommend universal adjuvant radiation therapy, even after gross-total resections, whereas others follow a wait-and-see strategy, even in selected patients with residual disease. To add to the complexity in managing these tumors, findings on postoperative imaging can often be deceiving, with small foci of residual or recurrent tumor being indistinguishable from postoperative changes.

In this report, we provide a prognostication scheme based on 2 independently prognostic factors, which accounts for a wide range of PFS in chordoma (cumulative hazard ratios ranging from 1 to 56.1 for PSFS, and from 1 to 75.6 for PSFR). As a general rule, every attempt is made to achieve a complete resection, and early MRI and reexploration/resection is always indicated if a complete resection is thought to be attainable. As such, after a maximal safe resection, 1p36 and 9p21 deletions could be used to further tailor subsequent management of a skull base chordoma.

The basic tenets of our proposed algorithm are presented in Fig. 3 and discussed below. According to this algorithm, tumors in group A (1p36 deletions $0 \%-15 \%$ and $9 \mathrm{p} 21$ dele- 


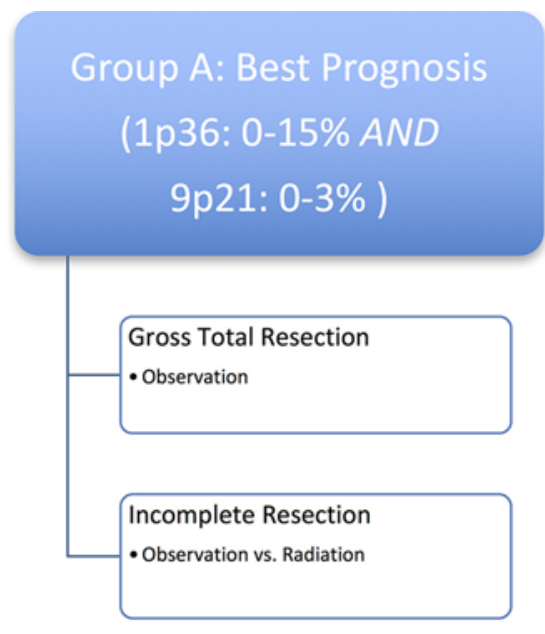

A

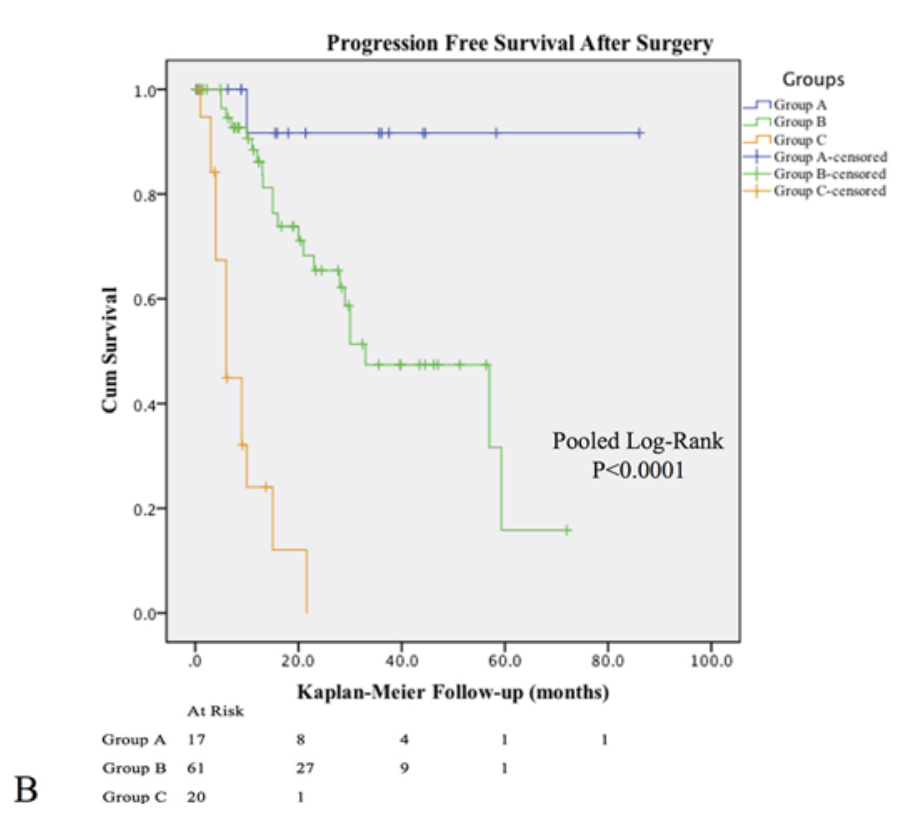

Group B: Intermediate Prognosis

\section{(all other combinations)}

\section{Gross Total Resection}

- Minimal concern for microscopic residual (e.g small tumor, negative margins)-> observation

- Any concern for microscopic residual -> radiation

\section{Incomplete Resection}

- Strong consideration for further surgery if a complete resection is possible without significant morbidity.

- Complete resection not possible $->$ radiation
Group C: Worst Prognosis

(1p36: >15\% AND

9p21: $25+\%$ )

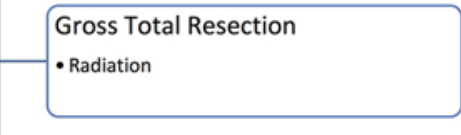

Incomplete Resection

- Strong consideration for further surgery to achieve a complete resection, even at the cost of some morbidity. Adjuvant radiation.

- Complete resection not possible -> radiation, and early consideration of experimental trials.

FIG. 3. A proposed management algorithm for clival chordoma based on the tumor's molecular panel. A: After resection of a clival chordoma, FISH for 1p36 deletions and homozygous 9p21 deletions can be used to guide management with regard to observation, radiotherapy, reoperation, or early consideration of experimental therapies. B: Kaplan-Meier curves showing overall PFSS (left) and PFSR (right) for each of the 3 groups. Figure is available in color online only.

tions $0 \%-3 \%$ ) are considered to have the best prognosis, and the best chances of control with radiation therapy. $\mathrm{Pa}$ tients with complete resections in this group could likely be observed closely, avoiding any potential morbidity from adjuvant radiation therapy. For patients in this group with residual tumors and for whom a complete resection cannot be attained without morbidity, adjuvant radiation is generally recommended. A survival benefit conferred by radiation has been repeatedly reported in the literature. $5-7,17,18,21,26$ Even though this was not replicated in our series for tumors in group $\mathrm{A}$, it is very possible that a selection bias or lack of statistical power may have made it harder for

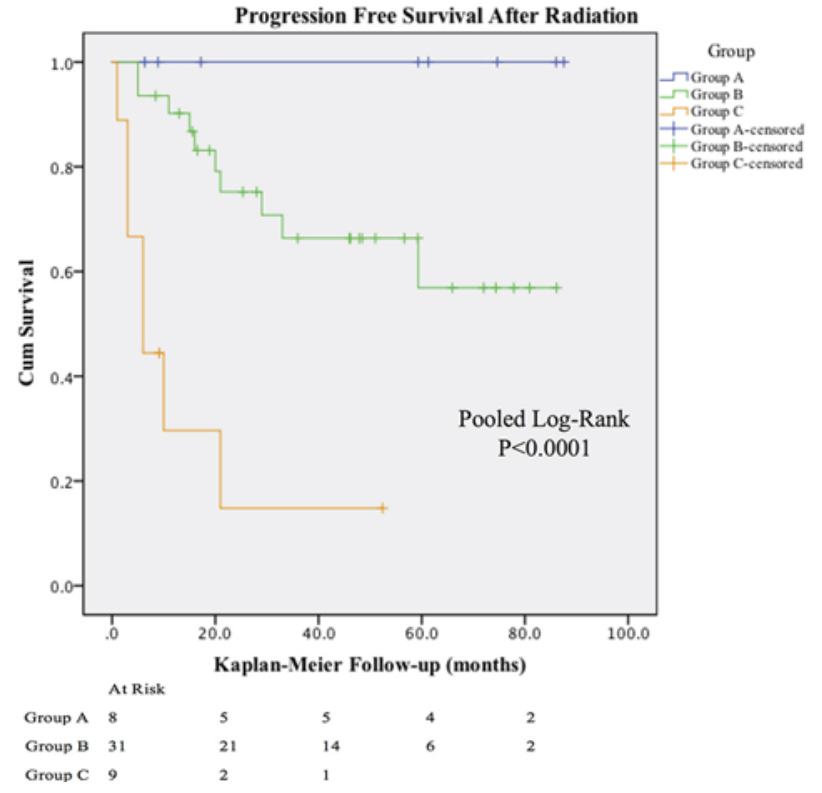

such a benefit to be revealed. However, the relatively long PFSS and PFSR in this group of tumors (median PFSS: 86 months, 36-month PFSS 92\%; median PFSR: 87 months, 36-month PFSR 100\% [Table 3]) may make close observation in selected patients a reasonable alternative. These include young men or women who want to have children and want to avoid the risk of hypopituitarism, very old patients, or patients who are absolutely averse to the idea of receiving radiation therapy. In such patients with a group $\mathrm{A}$ molecular profile, close observation may not be unreasonable, with further intervention reserved with the first signs of tumor progression. 
On the other end of the spectrum, tumors in group $\mathrm{C}$ (1p36 deletions $>15 \%$ and 9 p21 deletions $\geq 25 \%$ ) are associated with the worst prognosis, and are the least likely to be controlled with radiation therapy. Given the rapid progression that is expected from any residual tumor, adjuvant radiation therapy is likely universally indicated for this group. Aggressive multicorridor procedures to achieve a complete resection should be offered, and some morbidity (e.g., from bypassing/sacrificing vasculature or sacrificing a cranial nerve) may be acceptable if negative margins can be achieved. Conversely, if a complete resection is not possible, enrollment in experimental chemotherapeutic trials should be discussed early on, and the patient should be counseled appropriately.

Finally, group B (all other combinations) represents tumors with intermediate prognosis. For this group, withholding adjuvant radiation therapy may be reasonable after complete resections for which the concern for microscopic residual disease is low (e.g., after resection of a small tumor with no dural invasion and wide or negative margins). Alternatively, if there is any suspicion for microscopic remnants (as is usually the case) or there is gross residual tumor, adjuvant radiation therapy should be administered. In patients with incomplete resections, additional approaches should be offered prior to considering radiation therapy if a complete resection is felt to be feasible. However, the risk/ benefit ratio for these tumors likely does not warrant any significant approach-related morbidity to achieve a complete resection (e.g., sacrificing a cranial nerve).

Of course, many important factors, such as the patient's age and comorbidities, the patient's symptoms and functional capacity, and the patient's social support and belief systems, can influence clinical decision-making. As such, palliative surgical procedures aiming to relieve or prevent symptoms may always have a role, despite the patient's molecular profile and overall prognosis. It must be noted that the proposed management algorithm represents our way of applying the prognostication data and the general principles followed at our institution. Obviously, the prognostication information is free to interpretation and may lead to different management strategies by other practitioners. Whether the aforementioned algorithm indeed results in better overall outcomes will have to be determined prospectively. However, the proposed paradigm provides a basis for educated clinical decisions according to a tumor's predicted course, something that is currently lacking in the literature.

\section{Logistic Considerations}

Although modern prognostication panels are moving toward next-generation sequencing arrays accounting for a great variety of different genes, such data are currently not available for chordoma, and, given the difficulties with chordoma research, data validation allowing for clinical applicability of such panels is not expected to be available anytime soon. Conversely, the prognostication panel validated in this study is simple and easily accessible, while accounting for a wide range of prognosis in skull base chordomas.

\section{Limitations of the Current Study}

One limitation of this study is that not all tumors had the same extent of resection or the same timing/form of radiation therapy. As such, tumors with residuals may have been more likely to recur in a shorter interval. On the other hand, degree of resection can be subjective, and it is difficult to discern whether certain tumors' molecular profiles rendered them more invasive in the first place, leading to a subtotal resection. Given that further subclassification depending on the degree of resection or radiation modality would significantly hinder the statistical power of the study, such calculations were deferred at this time.

It must be noted that the proposed treatment algorithm has not been validated to improve outcomes, and merely represents our interpretation of the available prognostication data. Definitive conclusions cannot be made at this time about the optimal treatment strategy. However, with continued accumulation of additional data, subclassification of the prognostication profile with degrees of resection and timing/type of radiotherapy will likely provide enough statistical power to allow more definitive conclusions with regard to the management strategies.

Our comparisons of radiated versus not radiated tumors did not show a statistically significant difference in PFSR. However, there is a clear selection bias in patients selected to not receive radiation therapy and the number of these is low, significantly decreasing the statistical power of this portion of the analysis.

Another limitation is the use of the molecular profile of the tumors from the surgery preceding radiation therapy to evaluate PFSR. As the molecular profile of the tumors could change over time, the information used may not be entirely reflective of the true nature of the tumor at the time of progression. However, given that obtaining updated samples before radiation therapy is not realistic, the molecular profile from the resection preceding radiation therapy is pragmatically the best approximation.

In addition, although 1p36 and 9p21 deletions appear to play a significant role in the tumor's phenotypic mitotic activity from a statistical standpoint, their biological significance has to be further evaluated with functional tests in chordoma cell lines before such conclusions can be made.

\section{Conclusions}

To date, there is no reliable method of predicting the significant variability in behavior of skull base chordomas so as to guide clinical decision-making and patient education. The current report provides a validated prognostication scheme based on 2 independently prognostic molecular markers, 1p36 deletions and homozygous 9p21 deletions, which account for a wide spectrum of prognoses and response to radiation therapy. These markers allow the development of a management algorithm based on the tumor's predicted biological behavior, something that is currently missing in the literature. In addition, these results facilitate more informed patient education and could potentially help screen patients for appropriate chemotherapeutic trials.

\section{References}

1. Akhavan-Sigari R, Gaab MR, Rohde V, Abili M, Ostertag H: Expression of PDGFR- $\alpha$, EGFR and c-MET in spinal chordoma: a series of 52 patients. Anticancer Res 34:623-630, 2014 
2. Akhavan-Sigari R, Gaab MR, Rohde V, Abili M, Ostertag $\mathrm{H}$ : Prognostic significance of immunohistochemical expression of VEGFR2 and iNOS in spinal chordoma. Eur Spine $\mathbf{J}$ 23:2416-2422, 2014

3. Akhavan-Sigari R, Gaab MR, Rohde V, Brandis A, Tezval $\mathrm{H}$, Abili M, et al: Expression of vascular endothelial growth factor receptor 2 (VEGFR-2), inducible nitric oxide synthase (iNOS), and Ki-M1P in skull base chordoma: a series of 145 tumors. Neurosurg Rev 37:79-88, 2014

4. Bagchi A, Mills AA: The quest for the 1p36 tumor suppressor. Cancer Res 68:2551-2556, 2008

5. Boari N, Gagliardi F, Cavalli A, Gemma M, Ferrari L, Riva P, et al: Skull base chordomas: clinical outcome in a consecutive series of 45 patients with long-term follow-up and evaluation of clinical and biological prognostic factors. J Neurosurg 125:450-460, 2016

6. Bohman LE, Koch M, Bailey RL, Alonso-Basanta M, Lee JYK: Skull base chordoma and chondrosarcoma: influence of clinical and demographic factors on prognosis: a SEER analysis. World Neurosurg 82:806-814, 2014

7. Chambers KJ, Lin DT, Meier J, Remenschneider A, Herr M, Gray ST: Incidence and survival patterns of cranial chordoma in the United States. Laryngoscope 124:1097-1102, 2014

8. Chen C, Yang HL, Chen KW, Wang GL, Lu J, Yuan Q, et al: High expression of survivin in sacral chordoma. Med Oncol 30:529, 2013

9. Chen K, Mo J, Zhou M, Wang G, Wu G, Chen H, et al: Expression of PTEN and mTOR in sacral chordoma and association with poor prognosis. Med Oncol 31:886, 2014

10. de Castro CV, Guimaraes G, Aguiar S Jr, Lopes A, Baiocchi $\mathrm{G}$, da Cunha IW, et al: Tyrosine kinase receptor expression in chordomas: phosphorylated AKT correlates inversely with outcome. Hum Pathol 44:1747-1755, 2013

11. Duan Z, Shen J, Yang X, Yang P, Osaka E, Choy E, et al: Prognostic significance of miRNA-1 (miR-1) expression in patients with chordoma. J Orthop Res 32:695-701, 2014

12. Henrich KO, Schwab M, Westermann F: 1p36 tumor suppression-a matter of dosage? Cancer Res 72:6079-6088, 2012

13. Horbinski C, Oakley GJ, Cieply K, Mantha GS, Nikiforova $\mathrm{MN}$, Dacic S, et al: The prognostic value of Ki-67, p53, epidermal growth factor receptor, 1p36, 9p21, 10q23, and 17p13 in skull base chordomas. Arch Pathol Lab Med 134:1170 1176,2010

14. Jones PS, Aghi MK, Muzikansky A, Shih HA, Barker FG II, Curry WT Jr: Outcomes and patterns of care in adult skull base chordomas from the Surveillance, Epidemiology, and End Results (SEER) database. J Clin Neurosci 21:1490-1496, 2014

15. Kitamura Y, Sasaki H, Kimura T, Miwa T, Takahashi S, Kawase T, et al: Molecular and clinical risk factors for recurrence of skull base chordomas: gain on chromosome $2 p$, expression of brachyury, and lack of irradiation negatively correlate with patient prognosis. J Neuropathol Exp Neurol 72:816-823, 2013

16. Klingler L, Trammell R, Allan DG, Butler MG, Schwartz HS: Clonality studies in sacral chordoma. Cancer Genet Cytogenet 171:68-71, 2006

17. Koutourousiou M, Gardner PA, Tormenti MJ, Henry SL, Stefko ST, Kassam AB, et al: Endoscopic endonasal approach for resection of cranial base chordomas: outcomes and learning curve. Neurosurgery 71:614-625, 2012

18. Koutourousiou M, Snyderman CH, Fernandez-Miranda J, Gardner PA: Skull base chordomas. Otolaryngol Clin North Am 44:1155-1171, 2011

19. Le LP, Nielsen GP, Rosenberg AE, Thomas D, Batten JM, Deshpande V, et al: Recurrent chromosomal copy number alterations in sporadic chordomas. PLoS One 6:e18846, 2011

20. Longoni M, Orzan F, Stroppi M, Boari N, Mortini P, Riva P: Evaluation of $1 \mathrm{p} 36$ markers and clinical outcome in a skull base chordoma study. Neuro Oncol 10:52-60, 2008
21. Matloob SA, Nasir HA, Choi D: Proton beam therapy in the management of skull base chordomas: systematic review of indications, outcomes, and implications for neurosurgeons. Br J Neurosurg 30:382-387, 2016

22. Miozzo M, Dalprà L, Riva P, Volontà M, Macciardi F, Pericotti S, et al: A tumor suppressor locus in familial and sporadic chordoma maps to 1p36. Int J Cancer 87:68-72, 2000

23. Osaka E, Kelly AD, Spentzos D, Choy E, Yang X, Shen JK, et al: MicroRNA-155 expression is independently predictive of outcome in chordoma. Oncotarget 6:9125-9139, 2015

24. Romagosa C, Simonetti S, López-Vicente L, Mazo A, Lleonart ME, Castellvi J, et al: p16(Ink4a) overexpression in cancer: a tumor suppressor gene associated with senescence and high-grade tumors. Oncogene 30:2087-2097, 2011

25. von Witzleben A, Goerttler LT, Marienfeld R, Barth H, Lechel A, Mellert K, et al: Preclinical characterization of novel chordoma cell systems and their targeting by pharmocological inhibitors of the CDK4/6 cell-cycle pathway. Cancer Res 75:3823-3831, 2015

26. Walcott BP, Nahed BV, Mohyeldin A, Coumans JV, Kahle KT, Ferreira MJ: Chordoma: current concepts, management, and future directions. Lancet Oncol 13:e69-e76, 2012

27. Yakkioui Y, Temel Y, Creytens D, Jahanshahi A, Fleischeuer R, Santegoeds RGC, et al: A comparison of cell-cycle markers in skull base and sacral chordomas. World Neurosurg 82:e311-e318, 2014

28. Zhang K, Chen H, Wu G, Chen K, Yang H: High expression of SPHK1 in sacral chordoma and association with patients' poor prognosis. Med Oncol 31:247, 2014

29. Zou MX, Huang W, Wang XB, Li J, Lv GH, Wang B, et al: Reduced expression of miRNA-1237-3p associated with poor survival of spinal chordoma patients. Eur Spine J 24:17381746,2015

30. Zou MX, Huang W, Wang XB, Lv GH, Li J, Deng YW: Identification of miR-140-3p as a marker associated with poor prognosis in spinal chordoma. Int J Clin Exp Pathol 7:4877-4885, 2014

\section{Disclosures}

The authors report no conflict of interest concerning the materials or methods used in this study or the findings specified in this paper.

\section{Author Contributions}

Conception and design: Gardner, Zenonos. Acquisition of data: Zenonos. Analysis and interpretation of data: Gardner, Zenonos, Chang, Panayidou, Seethala. Drafting the article: Zenonos, Mukherjee. Critically revising the article: Gardner, Zenonos, Mukherjee, Snyderman, Fernandez-Miranda, Wang, Seethala. Reviewed submitted version of manuscript: Gardner, Zenonos, Mukherjee, Snyderman, Fernandez-Miranda, Wang, Seethala. Statistical analysis: Zenonos, Chang, Panayidou. Administrative/ technical/material support: Gardner, Zenonos, Fernandez-Miranda. Study supervision: Gardner, Zenonos, Snyderman, FernandezMiranda, Wang, Seethala.

\section{Supplemental Information \\ Online-Only Content}

Supplemental material is available with the online version of the article.

Supplementary Table 1. https://thejns.org/doi/suppl/10.3171/ 2018.3.JNS172321.

\section{Correspondence}

Paul A. Gardner: UPMC Presbyterian Hospital, Pittsburgh, PA. gardpa@upmc.edu. 